\title{
Mechanism for Limiting Thickness of Thin Oxide Films on Aluminum
}

\author{
Jakub D. Baran, Henrik Grönbeck, ${ }^{*}$ and Anders Hellman ${ }^{\dagger}$ \\ Department of Applied Physics and Competence Centre for Catalysis, Chalmers University of Technology, \\ SE-412 96 Göteborg, Sweden
}

(Received 12 September 2013; published 9 April 2014)

\begin{abstract}
A first-principles account of the observed limiting thickness of oxide films formed on aluminum during oxidizing conditions is presented. The results uncover enhanced bonding of oxygen to thin alumina films in contact with metallic aluminum that stems from charge transfer between a reconstructed oxide-metal interface and the adsorbed molecules. The first-principles results are compared with the traditional CabreraMott (CM) model, which is a classical continuum model. Within the CM model, charged surface oxygen species and metal ions generate a (Mott) potential that drives oxidation. An apparent limiting thickness is observed as the oxidation rate decreases rapidly with film growth. The present results support experimental estimates of the Mott potential and film thicknesses. In contrast to the CM model, however, the calculations reveal a real limiting thickness that originates from a diminishing oxygen adsorption energy beyond a certain oxide film thickness.
\end{abstract}

DOI: 10.1103/PhysRevLett.112.146103

Oxidation of metals has tremendous impact on society, and corrosion alone costs the U.S. trillions of dollars each year [1]. Under controlled conditions, however, oxidation plays an important role; thin oxides are used as catalysts, sensors, dielectrics, and corrosion inhibitors. For the latter purpose, metals such as aluminum and rhodium are preferred, as they develop protective oxide layers that inhibit bulk oxidation. Owing to its importance, there have been numerous experimental and theoretical efforts concerning the fundamental understanding of oxidation processes and, especially, the initial growth.

The seminal work of Cabrera and Mott (CM) of the mid20th century still remains the key theoretical model for growth of thin oxides on metals, see Fig. 1 [2]. According to $\mathrm{CM}$, electrons from the Fermi level of the metal substrate $\left(\epsilon_{F}\right)$ traverse the developing oxide film (with a band gap of $E_{g}$ ) by either tunneling or thermionic emission to acceptor levels $\left(\varphi_{a}\right)$ of oxygen species, thereby, forming different types of anions $\left(\mathrm{O}_{2}^{2-}, \mathrm{O}_{2}^{-}, \mathrm{O}^{2-}, \mathrm{O}^{-}\right)$on the oxide surface. The negative anions and the positive counterpart at the metal-oxide interface generate an electric potential, called the Mott potential $\left(V_{M}\right)$, which effectively lowers the energy barriers for migration of cations and/or anions through the oxide. This leads to a high oxidation rate even at low temperatures. As the oxide thickness increase, the additional effect of the Mott potential diminishes, and the oxidation process effectively stops at an apparent limiting thickness. It could be noted that although the original CM model discusses charge transfer through tunneling or thermionic emission, the actual transfer is probably defect or polaron mediated. However, this issue is not addressed in the present Letter.

Oxidation of aluminum is the prototypical process owing to the simple but versatile electronic structure of aluminum
PACS numbers: 68.35.bd, 68.43.Bc, 68.47.Gh, 81.15.Aa

when discussing thin film growth, and this system has played a central role in the establishment of the CM model $[3,4]$. Recently, Zhou et al. [5,6] showed that the thickness of the aluminum oxide on $\mathrm{Al}(111)$ can be tuned by the oxygen pressure. It was observed that the thickness of the alumina film increases with increasing oxygen pressure up



FIG. 1 (color online). (a) Schematic energy level diagram for oxide growth. The Fermi energy of the metal is denoted $\epsilon_{F}$, the band gap of the developing oxide $E_{g}$ and the adsorbate level $\varphi_{a}$. Anionic adsorbates are formed by charge transfer between the metal and the adsorbate. In this case, the adsorbate level is below the Fermi energy of the metal. (b) Thickness of oxide film as a function of time using experimentally derived values of system parameters for oxide growth on aluminum [2]. In the CM model, an apparent limiting thickness is reached after a few hours owing to the diminishing effect of the Mott potential as the film grows thicker. 
to 1 Torr, beyond which it remains constant at a thickness of $12.4 \AA$. The maximum Mott potential associated with the highest pressure was found to be $1.6 \mathrm{~V}$, which is in agreement with previous studies [2-4].

Although conceptually powerful, the CM model is based on a classical continuum description of the system and neither the actual atomic configuration nor the electronic structure are taken into account. These properties are accessible through first-principles quantum mechanical calculations. However, the complexity involved in the oxidation process together with the considerable system size (several hundreds of atoms) have hindered, so far, an atomistic assessment of the CM model. Previous efforts have concentrated on the oxide-metal interface [7-12] and initial growth [13-15]. In the present Letter, we investigate the underlying atomic and electronic effects that govern the thickness of the protective oxide layer on aluminum from first principles.

The calculations were performed within the density functional theory (DFT) with the Perdew-Burke-Ernzerhof [16] approximation for exchange-correlation energy as implemented in the Vienna ab initio simulation package (VASP) [17-19]. The projector augmented-wave method [20] is used with a plane-wave basis set energy cutoff of $400 \mathrm{eV}$. The total energies were extrapolated to $0 \mathrm{~K}$ from calculations where the Fermi distribution was smeared by a Gaussian function of $0.02 \mathrm{eV}$ [21]. Brillouin integration was performed using a $(2,2,1)$ Monkhorst-Pack mesh. The periodically replicated slabs were separated by a vacuum region of at least $20 \AA$ and a dipole correction was applied to avoid interactions between repeated slabs. All calculations were performed without any symmetry restrictions, and ionic relaxations were performed until atomic forces were less than $0.02 \mathrm{eV} / \AA$.

A large number of different atomic structures have been considered in order to capture the necessary chemical properties of aluminum oxidation. Details of the explored systems are described in the Supplemental Material [30]. As for aluminum protected by a thin oxide, we modeled this by an $\alpha-\mathrm{Al}_{2} \mathrm{O}_{3}(0001) / \mathrm{Al}(111)$ interface, where the oxide film was described by a $p(3 \times 3) \alpha-\mathrm{Al}_{2} \mathrm{O}_{3}$ slab with 2 to 11 layers, and the $\mathrm{Al}(111)$ was modeled by three layers of a $p(5 \times 5)$ hexagonal surface cell. The lattice misfit between $\alpha-\mathrm{Al}_{2} \mathrm{O}_{3}(0001)$ and $\mathrm{Al}(111)$ is only $0.9 \%$. The calculations show that an $\mathrm{Al}$ termination of the oxide, both at the surface and at the metal-oxide interface, is thermodynamically preferred for the $\alpha-\mathrm{Al}_{2} \mathrm{O}_{3}(0001)$ over the considered $\mathrm{O}_{2}$ pressure range. This is consistent with experiment $[22,23]$ as well as with previous DFT studies [11] including DFT molecular dynamics (MD) simulations of the $\alpha-\mathrm{Al}_{2} \mathrm{O}_{3} / \mathrm{Al}$ interface [12].

In Fig. 2(a), the adsorption energy per $\mathrm{O}_{2}$ molecule on $\mathrm{Al}_{2} \mathrm{O}_{3} / \mathrm{Al}(111)$ for different numbers of $\alpha-\mathrm{Al}_{2} \mathrm{O}_{3}$ layers and oxygen coverages is shown. There is a slow decrease in the adsorption energy as the film thickness increases.
Taking the example of $1 / 9$ coverage, the adsorption energy decreases from 2.98 to $0.08 \mathrm{eV}$ when the film thickness is increased from $2(\sim 4 \AA)$ to $11(\sim 22 \AA)$ layers. This should be compared with the calculated adsorption energy of $\mathrm{O}_{2}$ on bare $\mathrm{Al}(111)$ and unsupported $\mathrm{Al}_{2} \mathrm{O}_{3}(0001)$ which is calculated to be $7.56 \mathrm{eV}$ (dissociative adsorption) and $0.01 \mathrm{eV}$ (molecular adsorption), respectively. Thus, it is clear that molecular oxygen chemisorbs strongly up to a few layers and that the adsorption energy vanishes (resembling the physisorbed case of bulk alumina) at 11 layers of $\alpha-\mathrm{Al}_{2} \mathrm{O}_{3}(0001) . \mathrm{O}_{2}$ adsorbs preferably over undercoordinated aluminum sites, and there are nine such different sites in the considered surface cell. However, the adsorption energy varies moderately with surface site. For the case of five layers of $\alpha-\mathrm{Al}_{2} \mathrm{O}_{3}(0001)$ on $\mathrm{Al}(111)$, seven of the sites has an adsorption energy of $1.53 \mathrm{eV}(\sim 0.02 \mathrm{eV})$, whereas the remaining two sites have adsorption energies of 1.23 and $1.22 \mathrm{eV}$, respectively. The differences in adsorption energies between the sites originate from nonequivalent local atomic structure at the oxide-metal interface directly below the adsorbate. The calculated adsorption energy per $\mathrm{O}_{2}$ molecule at different coverages obeys a monotonic decrease with increasing $\mathrm{O}_{2}$ coverage. This yields a close to zero adsorption energy at full monolayer coverage for a nine layer thick alumina film.

As shown in Figs. 2(b) and 2(c), the strength of $\mathrm{O}_{2}$ adsorption is directly related to the amount of charge that is transferred to the molecule. For two layers of $\mathrm{Al}_{2} \mathrm{O}_{3}$, there is a charge transfer of almost two electrons, and the magnetic moment of the molecule is completely quenched, which indicates the formation of an $\mathrm{O}_{2}^{2-}$ anion. For four layers of alumina, there is a charge donation of one electron and the adsorbed oxygen contains one unpaired electron (formation of an $\mathrm{O}_{2}^{-}$anion). The general trend is a decrease in the charge transfer as the oxide film becomes thicker, which results in less quenching of the $\mathrm{O}_{2}$ magnetic moment and a decrease in the $\mathrm{O}-\mathrm{O}$ bond distance [see Fig. 2(d)]. The charge transfer and the $\mathrm{O}-\mathrm{O}$ bond distance have an interesting thickness dependence with a plateau for six to eight layers. This signals different regimes characterized by a charge transfer of two, one, and zero electrons to the $\mathrm{O}_{2}$ molecule. The plateau is not as pronounced in the calculated adsorption energies as this quantity is a consorted measure of interactions of the adsorbate with the alumina, the image charge between the charged adsorbate and the metal, and changes in the interactions at the metaloxide interface.

The initial atomic structure at the interface between the metal and the oxide (two layers) is shown in Fig. 2(e). The aluminum atoms that are in close proximity with the cation site of the oxide (which would be occupied if the alumina crystal continued to grow along the [0001] direction) are dragged out from the metal surface in the direction of this site. This rearrangement results in the formation of $\mathrm{Al}$ vacancies in the aluminum slab. The remaining $\mathrm{Al}$ atoms of 



FIG. 2 (color online). (a)-(d) Adsorption properties of $\mathrm{O}_{2}$ on $\mathrm{Al}_{2} \mathrm{O}_{3} / \mathrm{Al}(111)$ as a function of oxide trilayers. (a) Adsorption energy for four different coverages. (b) Magnetic moment of the system at a coverage of 1/9. (c) Calculated Bader charge on the adsorbed $\mathrm{O}_{2}$. (d) O-O bond distance of the adsorbed molecule. (e) and (f) show the atomic structure of the interface with and without adsorbed $\mathrm{O}_{2}$. Atomic color codes: Aluminum (blue/bright) and Oxygen (red/dark). (g) Gibbs free energy of $\mathrm{O}_{2}$ at a coverage of $1 / 9$ as a function of oxygen partial pressure at a temperature of $300 \mathrm{~K}$. The energies are given for different numbers of alumina layers.

the first metal layer are significantly buckled towards the oxide. The present results are in agreement with MD simulation of the $\alpha-\mathrm{Al}_{2} \mathrm{O}_{3} / \mathrm{Al}$ interface by Kang et al. [12], where it was found that the concentration of $\mathrm{Al}$ vacancies is close to $10 \%$. In our case, the concentration of clear $\mathrm{Al}$ vacancies is $\sim 12 \%$.

$\mathrm{O}_{2}$ adsorption results in pronounced structural reconstructions in the oxide film and the metal support. Generally, the aluminum atoms that bind to the oxide are pushed in the direction of the oxide. In the case of two $\mathrm{Al}_{2} \mathrm{O}_{3}$ layers [see Fig. 2(f)], $\mathrm{O}_{2}$ adsorption causes an upward displacement of the surface aluminum atom right below the adsorption site by $\sim 0.8 \AA$. This effect is less pronounced for thicker oxides.

The adsorption energy of molecules on metal supported ultrathin oxide film is a concerted phenomenon with several contributions, including the interaction with the image charge, structural relaxation at the adsorption site and relaxation at the metal-oxide interface. These contributions have been found to combine in a long-range bond mechanism [24-28] of electronegative or -positive adsorbates. The charge transfer is the most relevant contribution in connection to the $\mathrm{CM}$ model. However, the other contributions are also important for the enhancement of the adsorption energy. For instance, if no structural relaxation is allowed in the case of one $\mathrm{O}_{2}$ molecule adsorbed on four layers of $\alpha-\mathrm{Al}_{2} \mathrm{O}_{3}$ on $\mathrm{Al}(111)$, the adsorption energy is calculated to be only $0.44 \mathrm{eV}$. Thus, close to $3 / 4$ of the energetic enhancement originates from relaxations at the metal-oxide interface. We note that these contributions are only captured in an atomistic description of the system and are neglected in the CM model. This has important consequences for the analysis of experimental data within the $\mathrm{CM}$ model. The different contributions in the adsorption energy provide handles that can be used to enhance or prevent oxidation. It is, for example, possible to envision dopants at the interface that allow for a larger charge transfer (by modifying the work function) or a more substantial reconstruction (by lattice mismatch).

To facilitate the comparison with experiments $[5,6]$, the Gibbs free energy of $\mathrm{O}_{2}$ adsorption at $1 / 9$ coverage is shown as a function of pressure at room temperature in Fig. 2(g). The results reveal that $\mathrm{O}_{2}$ will adsorb at virtually any pressure on a five-layer oxide film. Hence, there is a clear thermodynamic driving force to form at least five layers of a protective oxide. This is in agreement with measurements showing spontaneous formation of an ultrathin oxide film even at very low temperatures [2]. From five layers, an increase in the $\mathrm{O}_{2}$ pressure results in adsorption on increasingly thicker films. However, at room temperature 
and atmospheric pressure, oxygen will not adsorb on films thicker than nine layers, which corresponds to $\sim 18 \AA$. As the oxygen adsorption is a prerequisite for further oxide growth, we identify physical and chemical reasons for the observed limiting thickness of $\mathrm{Al}_{2} \mathrm{O}_{3}$ grown on metallic aluminum. The present results show that this limit is reached around $18 \AA$. The calculated limiting thickness at 1 Torr is only slightly larger than the one recently measured by Zhou et al. $[5,6]$ The minor discrepancy (roughly one trilayer) can be attributed to the simplification of the structural models as well as to the well known overbinding of adsorption energies of $\mathrm{O}_{2}$ predicted by the current version of DFT [16]. It should be noted that the initially formed oxide layers in the experiment appear to be disordered [5], which only gradually transform into crystalline $\alpha-\mathrm{Al}_{2} \mathrm{O}_{3}$ [29]. However, we have explicitly confirmed that our conclusions are not critically dependent on the chosen phase of alumina or the presence of defects in the film [30].

Analysis of the calculated atomic charges allow for determination of the Mott potential associated with oxygen adsorption (see Fig. 3) as a function of the
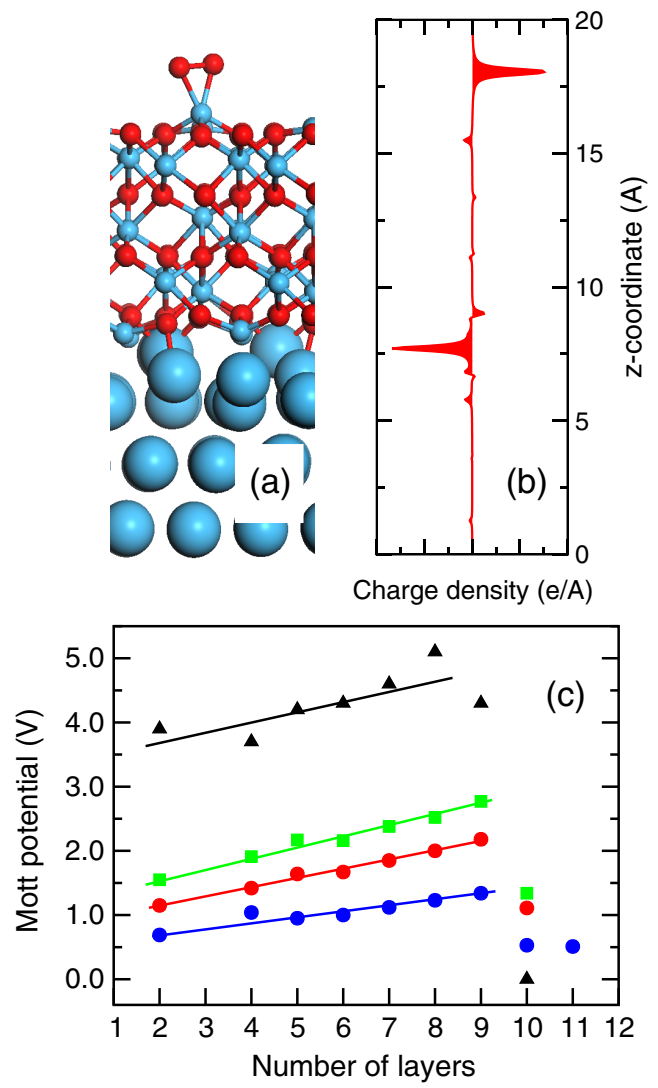

FIG. 3 (color online). (a) Optimized structure of $\mathrm{O}_{2}$ adsorbed on four trilayers of $\mathrm{Al}_{2} \mathrm{O}_{3}$ grown on $\mathrm{Al}(111)$. Color code as in Fig. 2. (b) Differences in Bader charges between $\mathrm{O}_{2} / \mathrm{Al}_{2} \mathrm{O}_{3} / \mathrm{Al}$ and $\mathrm{O}_{2}$ in the gas phase together with $\mathrm{Al}_{2} \mathrm{O}_{3} / \mathrm{Al}$. The charge differences are integrated in the $x y$ plane to give a profile along the $z$ axis. Charge is accumulated on $\mathrm{O}_{2}$ and depleted from the oxide-metal interface. (c) Mott potential as a function of number of layers and coverage. oxide thickness and oxygen coverage. The Mott potential is calculated from the Bader charges assuming a parallel plate capacitor charged with the oxygen's excess electrons. The Mott potential increases with increasing oxygen coverage from $\sim 1 \mathrm{~V}$ for $1 / 9 \mathrm{ML}$ to $\sim 4 \mathrm{~V}$ for a full oxygen monolayer. Although the value of potential increases slightly with increasing oxide thickness, the potential is fairly constant for a given oxygen coverage, which is in agreement with the CM model [2]. However, we calculate an abrupt drop of the potential for oxide thicknesses above $\sim 18 \AA$ (nine layers). The thickness for a vanishing Mott potential correlates with the absence of charge transfer, see Fig. 2(c).

Our first-principles calculations confirm a charge transfer process from the oxide-metal interface to the adsorbed oxygen, which is the key feature in the CM model. Moreover, the magnitude of the Mott potential agrees with experimental estimates $[2,5]$. It is interesting to note that, although there is a slow decrease of the Gibbs free energy of the adsorbed oxygen molecule, the Mott potential does not display such behavior. Instead, the first-principles derived Mott potential shows that in cases that oxygen can adsorb on the oxide, the charge transfer associated with this process is sufficient to generate a significant Mott potential. However, as the Gibbs free energy turns positive (beyond nine oxide layers at room temperature and $\sim 5$ Torr) the oxygen adsorption is prohibited and the oxidation process will terminate. Whereas the CM model predicts an apparent limiting thickness due to slow kinetics, the present results uncover a real limiting thickness due to low $\mathrm{O}_{2}$ adsorption energy. This is a completely new interpretation of the cause behind the observed limiting thickness on alumina and provides an explanation of the pressure dependence in film thickness recently observed experimentally [5].

To conclude, first-principles calculations support some of the key assumptions of the CM model. Based on our results, we estimated the limiting thickness of $\mathrm{Al}_{2} \mathrm{O}_{3}$ on $\mathrm{Al}(111)$ to about $18 \AA$, which agrees with experimental data. The charge transfer to adsorbed oxygen molecules will generate a Mott potential across the oxide sufficient to drive the oxidation process. However, the underlying reason for the observed limiting thickness is the decrease in adsorption energy of oxygen molecules that will prohibit the supply of oxygen. A secondary effect is the breakdown of the Mott potential when oxygen does not adsorb. This understanding provides physical and chemical handles on the oxidation process which will potentially have a large impact on industrial applications where corrosion is an important obstacle.

Support from the Swedish Research Council and the Chalmers Area of Advance Material and Nano is acknowledged. The calculations were performed at PDC (Stockholm) and C3SE (Göteborg). 
*Corresponding author. henrik.gronbeck@chalmers.se †Corresponding author.anders.hellman@chalmers.se

[1] http://www.g2mtlabs.com/2011/06/nace-cost-of-corrosionstudy-update/.

[2] N. Cabrera and N.F. Mott, Rep. Prog. Phys. 12, 163 (1949).

[3] F. P. Fehlner and N. F. Mott, Oxid. Met. 2, 59 (1970).

[4] A. Atkinson, Rev. Mod. Phys. 57, 437 (1985).

[5] N. Cai, G. Zhou, K. Müller, and D. E. Starr, Phys. Rev. Lett. 107, 035502 (2011).

[6] N. Cai, G. Zhou, K. Müller, and D. E. Starr, Phys. Rev. B 84, 125445 (2011).

[7] M. Vermeersch, F. Malengreau, R. Sporken, and R. Caudano, Surf. Sci. 323, 175 (1995).

[8] D. R. Jennison, C. Verdozzi, P. A. Schultz, and M. P. Sears, Phys. Rev. B 59, R15605 (1999).

[9] L. P. H. Jeurgens, W. G. Sloof, F. D. Tichelaar, and E. J. Mittemeijer, Phys. Rev. B 62, 4707 (2000).

[10] D. R. Jennison and A. Bogicevic, Surf. Sci. 464, 108 (2000).

[11] D. J. Siegel, L. G. Hector, and J. B. Adams, Phys. Rev. B 65, 085415 (2002).

[12] J. Kang, J. Zhu, C. Curtis, D. Blake, G. Glatzmaier, Y. H. Kim, and S. H. Wei, Phys. Rev. Lett. 108, 226105 (2012).

[13] I. Popova, V. Zhukov, and J. J. T. Yates, Phys. Rev. Lett. 89, 276101 (2002).

[14] A. Hellman, B. Razaznejad, Y. Yourdshahyan, H. Ternow, I. Zoric, and B. I. Lundqvist, Surf. Sci. 532-535, 126 (2003).

[15] J. Behler, B. Delley, S. Lorenz, K. Reuter, and M. Scheffler, Phys. Rev. Lett. 94, 036104 (2005).

[16] J. P. Perdew, K. Burke, and M. Ernzerhof, Phys. Rev. Lett. 77, 3865 (1996).

[17] G. Kresse and J. Hafner, Phys. Rev. B 49, 14251 (1994).

[18] G. Kresse and J. Furthmuller, Phys. Rev. B 54, 11169 (1996).

[19] G. Kresse and D. Joubert, Phys. Rev. B 59, 1758 (1999).

[20] P. E. Blochl, Phys. Rev. B 50, 17953 (1994).

[21] The smearing of the Fermi distribution with respect to method and amount of smearing for the multicomponent system (metal-oxide-molecule) were carefully tested. Besides Gaussian smearing, 1st and 2nd order MethfesselPaxton smearing with a width of 0.02 and $0.2 \mathrm{eV}$ together with Fermi smearing of width $0.02 \mathrm{eV}$ were investigated. Only minor changes (less than $0.1 \mathrm{eV}$ ) in the adsorption energy was obtained.

[22] D. L. Medlin, K. F. McCarthy, R. Q. Hwang, S. E. Guthrie, and M. I. Baskes, Thin Solid Films 299, 110 (1997).
[23] S. H. Oh, M. F. Chisholm, Y. Kauffmann, W. D. Kaplan, M. Rühle, and C. Scheu, Science 330, 489 (2010).

[24] G. Pacchioni, L. Giordano, and M. Baistrocchi, Phys. Rev. Lett. 94, 226104 (2005).

[25] H. Grönbeck, J. Phys. Chem. B 110, 11977 (2006).

[26] A. Hellman and H. Grönbeck, Phys. Rev. Lett. 100, 116801 (2008).

[27] P. Frondelius, A. Hellman, K. Honkala, H. Häkkinen, and H. Grönbeck, Phys. Rev. B 78, 085426 (2008).

[28] H. J. Freund and G. Pacchioni, Chem. Soc. Rev. 37, 2224 (2008).

[29] L. P. H. Jeurgens, W. G. Sloof, F. D. Tichelaar, and E. J. Mittmeijer, J. Appl. Phys. 92, 1649 (2002).

[30] See Supplemental Material (SM) at http://link.aps.org/ supplemental/10.1103/PhysRevLett.112.146103 for details of the considered systems, which includes Refs. [31-40]. Adsorption of $\mathrm{O}_{2}$ on $\gamma-\mathrm{Al}_{2} \mathrm{O}_{3}$ films supported on $\mathrm{Al}(111)$ were performed for four $(\sim 8 \AA)$ and eight $(\sim 16 \AA)$ layers of $\gamma-\mathrm{Al}_{2} \mathrm{O}_{3}$. The corresponding adsorption energies are 1.60 and $0.82 \mathrm{eV}$, respectively. Thus, very close to the $\alpha-\mathrm{Al}_{2} \mathrm{O}_{3}$ values. Calculations with a defect containing film (see SM) were performed with one $\mathrm{O}_{2}$ molecule adsorbed on a four layer $\alpha-\mathrm{Al}_{2} \mathrm{O}_{3}(0001)$ on $\mathrm{Al}(111)$. The adsorption energy was calculated to be $1.38,1.30$, and $1.83 \mathrm{eV}$ for one $\mathrm{Al}$ defect at the interface, one $\mathrm{O}$ removed from the oxide, and two $\mathrm{O}$ removed from the oxide, respectively. The adsorption energy of $\mathrm{O}_{2}$ on this system without defects is $1.80 \mathrm{eV}$.

[31] R. D. Johanson, NIST Computational Chemistry Comparison and Benchmark Database; NIST Standard Reference Database, August 2011.

[32] Y.S. Touloukian, Thermophysical Properties of Matter (Plenum, New York, 1975), Vol. 12.

[33] Y. Yourdshahyan, B. Razaznejad, and B. I. Lundqvist, Phys. Rev. B 65, 075416 (2002).

[34] X. G. Wang, A. Chaka, and M. Scheffler, Phys. Rev. Lett. 84, 3650 (2000).

[35] C. Ruberto, Y. Yourdshahyan, and B. I. Lundqvist, Phys. Rev. B 67, 195412 (2003).

[36] B. Hinnemann and E. A. Carter, J. Phys. Chem. C 111, 7105 (2007).

[37] A. Hellman and H. Grönbeck, J. Phys. Chem. C 113, 3674 (2009).

[38] R. W. G. Wyckoff, Crystal Structures (Interscience Publishers, New York, 1963), Vol. 2.

[39] R. H. French, J. Am. Ceram. Soc. 73, 477 (1990).

[40] M. Digne, P. Sautet, P. Raybaud, P. Euzen, and H. Toulhoat, J. Catal. 226, 54 (2004). 